\title{
NONDEFECTIVE STATIONARY DISCS AND 2-JET DETERMINATION IN HIGHER CODIMENSION
}

\author{
FLORIAN BERTRAND AND FRANCINE MEYLAN
}

\begin{abstract}
We discuss the links between stationary discs, the defect of analytic discs, and 2-jet determination of CR automorphisms of generic nondegenerate real submanifolds of $\mathbb{C}^{N}$ of class $\mathcal{C}^{4}$.
\end{abstract}

\section{INTRODUCTION}

In his important paper [29], Tumanov introduced the notion of defect of an analytic disc $f$ attached to a generic submanifold $M \subset \mathbb{C}^{N}$, which was defined equivalently by Baouendi, Rothschild and Trépreau [3] as the dimension of the real vector space of all holomorphic lifts $\boldsymbol{f}$ in $T^{*}\left(\mathbb{C}^{N}\right)$ of $f$ attached to the conormal bundle $N^{*}(M)$. In particular, Tumanov proved that the existence of nondefective analytic discs, that is of defect 0 , attached to $M$ implies the wedge extendability of CR functions. In the present paper, we introduce a new notion of nondegeneracy of the Levi map which expresses the existence of a nondefective stationary disc attached to the quadric model of $M$. Using a deformation argument developed by Forstnerič [15] and Globevnik [16], we produce a family of stationary discs near that nondefective disc, which are uniquely determined by their 1-jet and which cover an open set of $M$. As an application of this theory, we deduce a 2-jet determination for CR automorphisms of our generic submanifold $M$.

Theorem 0.1. Let $M \subset \mathbb{C}^{N}$ be a $\mathcal{C}^{4}$ generic real submanifold. We assume that $M$ is $\mathfrak{D}$-nondegenerate at $p \in M$. Then any germ at $p$ of $C R$ automorphism of $M$ of class $\mathcal{C}^{3}$ is uniquely determined by its 2-jet at $p$.

We refer to Definition 1.3 for the notion of $\mathfrak{D}$-nondegenerate submanifold; this notion is closely related to the existence of a nondefective stationary disc (see Lemma 2.3). In the previous paper by the authors and Blanc-Centi [6], 2-jet determination is obtained under the more restrictive assumption that $M$ is fully nondegenerate (see Definition 1.2 in [6]). Indeed, while fully nondegeneracy imposes the codimension restriction $d \leq n$, $\mathfrak{D}$-nondegeneracy requires $d \leq 2 n$. For instance, the quadric

$$
\left\{\begin{array}{l}
\Re e w_{1}=\left|z_{1}\right|^{2} \\
\Re e w_{2}=\left|z_{2}\right|^{2} \\
\Re e w_{3}=z_{1} \overline{z_{2}}+\overline{z_{1}} z_{2}
\end{array}\right.
$$

and its perturbations are $\mathfrak{D}$-nondegenerate but not fully nondegenerate.

Stationary discs were introduced by Lempert [23] in his work on the Kobayashi metric of strongly convex domains and studied further in [17, 26, 30, 27]. Their use in the finite jet determination problem in the framework of finitely smooth submanifolds has been recently developed by several authors [5, 7, 6, 31. Finite jet determination problems for 
real analytic or $\mathcal{C}^{\infty}$ real submanifolds has attracted considerable attention. We know from results of Cartan [11, Tanaka 28, Chern and Moser [9], that germs of CR automorphism of a real-analytic Levi nondegenerate hypersurface $M$ in $\mathbb{C}^{N}$ are uniquely determined by their 2-jet at $p \in M$. We also refer for instance to the articles of Zaitsev [33], Baouendi, Ebenfelt and Rothschild [1], Baouendi, Mir and Rothschild [2], Ebenfelt, Lamel and Zaitsev [14, Lamel and Mir, 222, Juhlin [18, Juhlin and Lamel [19], Mir and Zaitsev [25] in the real analytic framework. We point out the works of Ebenfelt [12], Ebenfelt and Lamel [13, and Kim and Zaitsev [20], Kolar, Zaitsev and the third author [21], in the $\mathcal{C}^{\infty}$ setting.

During the completion of this work, we received a preprint by Tumanov [31], where 2jet determination of $\mathrm{CR}$ automorphisms is obtained for $\mathcal{C}^{4}$ generic strongly pseudoconvex Levi generating submanifold. His approach is based on the stationary discs method in the jet determination problem [5, 7, 6, and although apparently similar, his result and ours are in different settings of generic submanifolds of higher codimension. For instance, the quadric

$$
\left\{\begin{array}{l}
\Re e w_{1}=\left|z_{1}\right|^{2}-\left|z_{2}\right|^{2} \\
\Re e w_{2}=\left|z_{3}\right|^{2}
\end{array}\right.
$$

is $\mathfrak{D}$-nondegenerate but not strictly pseudoconvex. Nevertheless, the result of Tumanov and ours are a step towards the settlement of the following conjecture:

Conjecture. Consider a $\mathcal{C}^{4}$ generic real submanifold $M$ Levi nondegenerate in the sense of Tumanov and admitting a nondefective stationary disc passing through $p \in M$. Then any germ at $p$ of $C R$ automorphism of $M$ of class $\mathcal{C}^{3}$ is uniquely determined by its 2-jet $p$.

We would like to point out that, recently, the second author [24] gave an example of a quadric in $\mathbb{C}^{9}$ of finite type with 2 the only Hörmander number and Levi nondegenerate in the sense of Tumanov, for which the 2-jet determination for biholomorphisms does not hold. Therefore, even in the real analytic case, the question of an optimal bound for the jet determination for nondegenerate (in the sense of Definition 1.1) submanifolds remains open.

The paper is organized as follows. In Section 1 and Section 2, we introduce the setting of the work and the relevant notions; in particular we connect $\mathfrak{D}$-nondegeneracy with the construction of a nondefective stationary disc (Lemma 2.3). In Section 2, we first recall the main result on the existence of stationary discs (Theorem 3.1) and we prove that stationary discs near an initial nondefective disc are uniquely determined by their 1-jet (Proposition 3.3) and cover an open set in the conormal bundle (Proposition 3.4). This last result is particularly important and differs with the usual results in the theory, where it is proved that centers of such discs cover an open sets in either $T^{*} \mathbb{C}^{N}$ or $C^{N}$. Finally we prove Theorem 0.1 . The paper also contains an appendix on a more general result on jet determination for stationary discs. 


\section{Preliminaries}

We denote by $\Delta$ the unit disc in $\mathbb{C}$, by $\partial \Delta$ its boundary, and by $\mathbb{B} \subset \mathbb{C}^{N}$ the unit ball in $\mathbb{C}^{N}$.

1.1. Nondegenerate generic real submanifolds. Let $M \subset \mathbb{C}^{N}$ be a $\mathcal{C}^{4}$ generic real submanifold of real codimension $d \geq 1$ through $p$. After a local biholomorphic change of coordinates, we may assume that $p=0$ and that $M \subset \mathbb{C}^{N}=\mathbb{C}_{z}^{n} \times \mathbb{C}_{w}^{d}$ is given locally by the following system of equations

$$
\left\{\begin{aligned}
r_{1} & =\Re e w_{1}-{ }^{t} \bar{z} A_{1} z+O(3)=0 \\
& \vdots \\
r_{d} & =\Re e w_{d}-{ }^{t} \bar{z} A_{d} z+O(3)=0
\end{aligned}\right.
$$

where $A_{1}, \ldots, A_{d}$ are Hermitian matrices of size $n$. In the remainder $\mathrm{O}(3), z$ is of weight one and $\Im m w$ of weight two. We set $r=\left(r_{1}, \ldots, r_{d}\right)$.

We recall the following biholomorphic invariant notions introduced by Beloshapka in [4] and by the authors and Blanc-Centi in [6] that coincide in the hypersurface case and in case $N=4$.

Definition 1.1 (Beloshapka [4]). A $\mathcal{C}^{4}$ generic real submanifold $M$ of $\mathbb{C}^{N}$ of codimension $d$ and given by (1.1) is Levi nondegenerate at 0 in the sense of Beloshapka if the following two conditions are both satisfied

(a) $A_{1}, \ldots, A_{d}$ are linearly independent (equivalently on $\mathbb{R}$ or $\mathbb{C}$ ),

(b) $\bigcap_{j=1}^{d} \operatorname{Ker} A_{j}=\{0\}$.

Definition $1.2([6])$. A $\mathcal{C}^{4}$ generic real submanifold $M$ of $\mathbb{C}^{N}$ of codimension $d$ given by (1.1) is fully nondegenerate at 0 if the following two conditions are satisfied

(t) there exists a real linear combination $A=\sum_{j=1}^{d} c_{j} A_{j}$ that is invertible.

(f) there exists $V \in \mathbb{C}^{n}$ such that if we set $D$ to be the $n \times d$ matrix whose $j^{\text {th }}$ column is $A_{j} V$, and $B={ }^{t} \bar{D} A^{-1} D$, then $B$ and $\Re e B$ are invertible.

We point out that condition $(\mathfrak{a})$ implies a restriction on the codimension, namely $d \leq n^{2}$, and that condition $(\mathfrak{f})$ implies $d \leq n$. In the present paper, we introduce a less stringent notion of nondegeneracy by removing the assumption on the invertibility of $B$, which will allow us to work with codimension $d \leq 2 n$.

Definition 1.3. A $\mathcal{C}^{4}$ generic real submanifold $M$ of $\mathbb{C}^{N}$ of codimension $d$ given by (1.1) is $\mathfrak{D}$-nondegenerate at 0 if the following two conditions are satisfied

(t) there exists a real linear combination $A=\sum_{j=1}^{d} c_{j} A_{j}$ that is invertible.

(d) there exists $V \in \mathbb{C}^{n}$ such that if we set $D$ to be the $n \times d$ matrix whose $j^{\text {th }}$ column is $A_{j} V$, and $B={ }^{t} \bar{D} A^{-1} D$, then $\Re e B$ is invertible. 
We point out that condition ( $\mathfrak{d})$ implies that $\operatorname{span}_{\mathbb{R}}\left\{A_{1} V, \ldots, A_{d} V\right\}$ is of dimension $d$, and the converse holds in the strictly pseudoconvex case, that is, when $A$ is positive definite. Recall that condition (t) was introduced by Tumanov [30] and is essential for the construction of stationary disc; we say that $M$ is Levi nondegenerate at 0 in the sense of Tumanov if it satisfies conditon $(\mathfrak{t})$.

1.2. Spaces of functions. We introduce the spaces of functions we need. Let $k \geq 0$ be an integer and let $0<\alpha<1$. We denote by $\mathcal{C}^{k, \alpha}=\mathcal{C}^{k, \alpha}(\partial \Delta, \mathbb{R})$ the space of real-valued functions defined on $\partial \Delta$ of class $\mathcal{C}^{k, \alpha}$. The space $\mathcal{C}^{k, \alpha}$ is endowed with its usual norm

$$
\|f\|_{\mathcal{C}^{k, \alpha}}=\sum_{j=0}^{k}\left\|f^{(j)}\right\|_{\infty}+\sup _{\zeta \neq \eta \in \partial \Delta} \frac{\left\|f^{(k)}(\zeta)-f^{(k)}(\eta)\right\|}{|\zeta-\eta|^{\alpha}}
$$

where $\left\|f^{(j)}\right\|_{\infty}=\max _{\partial \Delta}\left\|f^{(j)}\right\|$. We set $\mathcal{C}_{\mathbb{C}}^{k, \alpha}=\mathcal{C}^{k, \alpha}+i \mathcal{C}^{k, \alpha}$ and we equip this space with the norm

$$
\|f\|_{\mathcal{C}_{\mathbb{C}}^{k, \alpha}}=\|\Re e f\|_{\mathcal{C}^{k, \alpha}}+\|\Im m f\|_{\mathcal{C}^{k, \alpha}} .
$$

We denote by $\mathcal{A}^{k, \alpha}$ the subspace of analytic discs in $\mathcal{C}_{\mathbb{C}}^{k, \alpha}$ consisting of functions $f: \bar{\Delta} \rightarrow$ $\mathbb{C}$, holomorphic on $\Delta$ with trace on $\partial \Delta$ belonging to $\mathcal{C}_{\mathbb{C}}^{k, \alpha}$. We now denote by $\mathcal{A}_{0}^{1, \alpha}$ the subspace of $\mathcal{C}_{\mathbb{C}}^{1, \alpha}$ of functions of the form $(1-\zeta) f$, with $f \in \mathcal{A}^{1, \alpha}$ and we equip $\mathcal{A}_{0}^{1, \alpha}$ with the norm

$$
\|(1-\zeta) f\|_{\mathcal{A}_{0}^{1, \alpha}}=\|f\|_{\mathcal{C}_{\mathbb{C}}^{1, \alpha}}
$$

which makes it a Banach space and isomorphic to $\mathcal{A}^{1, \alpha}$. We also denote by $\mathcal{C}_{0}^{1, \alpha}$ the subspace of $\mathcal{C}^{1, \alpha}$ of functions of the form $(1-\zeta) v$ with $v \in \mathcal{C}_{\mathbb{C}}^{1, \alpha}$. The space $\mathcal{C}_{0}^{1, \alpha}$ is equipped with the norm

$$
\|(1-\zeta) f\|_{\mathcal{C}_{0}^{1, \alpha}}=\|f\|_{\mathcal{C}_{\mathbb{C}}^{1, \alpha}}
$$

Note that $\mathcal{C}_{0}^{k, \alpha}$ is a Banach space.

\section{Stationary Discs AND THE DEFECT}

2.1. Stationary discs. Let $M$ be a $\mathcal{C}^{4}$ generic real submanifold of $\mathbb{C}^{N}$ of codimension $d$ given by (1.1). An analytic disc $f \in\left(\mathcal{A}^{k, \alpha}\right)^{N}$ is attached to $M$ if $f(\partial \Delta) \subset M$. Following Lempert [23] and Tumanov [30] we define

Definition 2.1. A holomorphic disc $f: \Delta \rightarrow \mathbb{C}^{N}$ continuous up to $\partial \Delta$ and attached to $M$ is stationary for $M$ if there exists a holomorphic lift $\boldsymbol{f}=(f, \tilde{f})$ of $f$ to the cotangent bundle $T^{*} \mathbb{C}^{N}$, continuous up to $\partial \Delta$ and such that for all $\zeta \in \partial \Delta, \boldsymbol{f}(\zeta) \in \mathcal{N} M(\zeta)$ where

$$
\mathcal{N} M(\zeta):=\left\{(z, w, \tilde{z}, \tilde{w}) \in T^{*} \mathbb{C}^{N} \mid(z, w) \in M,(\tilde{z}, \tilde{w}) \in \zeta N_{(z, w)}^{*} M \backslash\{0\}\right\},
$$

and where

$$
N_{(z, w)}^{*} M=\operatorname{span}_{\mathbb{R}}\left\{\partial r_{1}(z, w), \ldots, \partial r_{d}(z, w)\right\}
$$

is the conormal fiber at $(z, w)$ of $M$. The set of these lifts $\boldsymbol{f}=(f, \tilde{f})$, with $f$ non-constant, is denoted by $\mathcal{S}(M)$. 
Note that equivalently, an analytic disc $f \in\left(\mathcal{A}^{k, \alpha}\right)^{N}$ attached to $M$ is stationary for $M$ if there exists $d$ real valued functions $c_{1}, \ldots, c_{d}: \partial \Delta \rightarrow \mathbb{R}$ such that $\sum_{j=1}^{d} c_{j}(\zeta) \partial r_{j}(0) \neq 0$ for all $\zeta \in \partial \Delta$ and such that the map

$$
\zeta \mapsto \zeta \sum_{j=1}^{d} c_{j}(\zeta) \partial r_{j}(f(\zeta), \overline{f(\zeta)})
$$

defined on $\partial \Delta$ extends holomorphically on $\Delta$.

The set of such small discs is invariant under CR automorphisms. Recall the following essential result due to Webster [32] in the hypersurface case and to Tumanov [30] in higher codimension case.

Proposition $2.2([30])$. A generic real submanifold $M$ given by (1.1) satisfies $(\mathfrak{t})$ if and only the conormal bundle $N^{*} M$ is totally real at $\left(0, \sum_{j=1}^{d} c_{j} \partial r_{j}(0)\right)$, where the $c_{1}, \ldots, c_{d}$ are such that $\sum_{j=1}^{d} c_{j} A_{j}$ is invertible.

2.2. Nondefective stationary discs. We recall the notion of defect of an analytic disc, and more precisely of a stationary disc. Following [3, we say that a stationary disc $f$ is defective if it admits a lift $\boldsymbol{f}=(f, \tilde{f}): \Delta \rightarrow T^{*} \mathbb{C}^{N}$ such that $1 / \zeta . \boldsymbol{f}=(f, \tilde{f} / \zeta)$ is holomorphic on $\Delta$. The disc is nondefective if it is not defective. We investigate the notion of defective disc in case of a quadric. Consider a quadric submanifold $Q \subset \mathbb{C}^{N}=$ $\mathbb{C}_{z}^{n} \times \mathbb{C}_{w}^{d}$ of real codimension $d$ given by

$$
\left\{\begin{array}{c}
\rho_{1}=\Re e w_{1}-{ }^{t} \bar{z} A_{1} z=0 \\
\quad \vdots \\
\rho_{d}=\Re e w_{d}-{ }^{t} \bar{z} A_{d} z=0
\end{array}\right.
$$

where $A_{1}, \ldots, A_{d}$ are hermitian matrices of size $n$. We set $\rho=\left(\rho_{1}, \ldots, \rho_{d}\right)$. In that context, a stationary disc $f$ for $Q$ is defective if there exists $c=\left(c_{1}, \ldots, c_{d}\right) \in \mathbb{R}^{d} \backslash$ $\{0\}$ such that the map $\zeta \mapsto c \partial_{z} \rho(f(\zeta))=\sum_{j=1}^{d} c_{j} \partial_{z} \rho_{j}(f(\zeta))$ defined on $\partial \Delta$ extends holomorphically on $\Delta$. The latter relates with Tumanov's equivalent definition of the defect in [29]. In [6], we considered a special family of lifts $\boldsymbol{f}=(h, g, \tilde{h}, \tilde{g}) \in \mathcal{S}(Q)$ of the form

$$
\boldsymbol{f}=\left((1-\zeta) V, 2(1-\zeta)^{t} \bar{V} A_{1} V, \ldots, 2(1-\zeta)^{t} \bar{V} A_{d} V,(1-\zeta)^{t} \bar{V} A, \frac{\zeta}{2} c\right)
$$

where $V \in \mathbb{C}^{n}$ and $A:=\sum_{j=1}^{d} c_{j} A_{j}$. The following lemma is a crucial observation, according to which, condition $(\mathfrak{d})$ in Definition 1.3 implies the existence of a nondefective stationary disc of the form (2.2).

Lemma 2.3. A stationary disc $f=\left((1-\zeta) V, 2(1-\zeta)^{t} \bar{V} A_{1} V, \ldots, 2(1-\zeta)^{t} \bar{V} A_{d} V\right)$, where $V \in \mathbb{C}^{n}$, for $Q$ is nondefective if and only if $A_{1} V, \ldots, A_{d} V$ are $\mathbb{R}$-linearly independent. 
Proof. Let $\boldsymbol{f}$ be a stationary disc of the form (2.2) and assume that $\sum_{j=1}^{d} c_{j} A_{j} V=0$ for $c=\left(c_{1}, \ldots, c_{d}\right) \in \mathbb{R}^{d} \backslash\{0\}$. The disc

$$
\boldsymbol{f}=\left((1-\zeta) V, 2(1-\zeta){ }^{t} \bar{V} A_{1} V, \ldots, 2(1-\zeta)^{t} \bar{V} A_{d} V, 0, \frac{\zeta}{2} c\right)
$$

is a holomorphic lift of $f$, and thus, $f$ is defective.

Conversely, assume that $f=\left((1-\zeta) V, 2(1-\zeta)^{t} \bar{V} A_{1} V, \ldots, 2(1-\zeta)^{t} \bar{V} A_{d} V\right)$ is defective. It follows that there exists $c=\left(c_{1}, \ldots, c_{d}\right) \in \mathbb{R}^{d} \backslash\{0\}$ such that the map

$$
\sum_{j=1}^{d} c_{j} \partial_{z} \rho_{j}(f(\zeta))=(\bar{\zeta}-1) \sum_{j=1}^{d} c_{j}{ }^{t} \bar{V} A_{j}=(\bar{\zeta}-1) \sum_{j=1}^{d} c_{j}{ }^{t} \overline{\left(A_{j} V\right)}
$$

extends holomorphically on $\Delta$, which can only occur if $\sum_{j=1}^{d} c_{j} t \overline{A_{j} V}=0$.

Remark 2.4. Note that in the above, we do not assume that $M$ satsifies $(\mathfrak{t})$.

\section{NondefECTIVE STATIONARY DisCS AND JET DETERMinAtion}

We consider a quadric submanifold $Q \subset \mathbb{C}^{N}$ of real codimension $d$ defined by $\{\rho=0\}$, where $\rho$ is given by (2.1), satisfying $(\mathfrak{t})$ and an initial lift of a stationary disc $\boldsymbol{f}_{\mathbf{0}}=$ $\left(h_{0}, g_{0}, \tilde{h_{0}}, \tilde{g_{0}}\right)$ of the form (2.2) where $c_{1}, \ldots, c_{d}$ are chosen such that the matrix $A=$ $\sum_{j=1}^{d} c_{j} A_{j}$ is invertible. We define the affine space $\mathcal{A} \ni \boldsymbol{f}_{\mathbf{0}}$ to be the subset of $\left(\mathcal{A}^{1, \alpha}\right)^{2 N}$ of discs whose value at $\zeta=1$ is $(0,0,0, c / 2)$, that is, of discs of the form

$$
\left((1-\zeta) h,(1-\zeta) g,(1-\zeta) \tilde{h},(1-\zeta) \tilde{g}+\frac{\zeta}{2} c\right)
$$

We set

$$
\mathcal{S}_{0}(M)=\mathcal{S}(M) \cap \mathcal{A} \ni \boldsymbol{f}_{0}
$$

Let $G(\zeta)$ be the square matrix of size $2 N$ given by

$$
G(\zeta)=\left(\begin{array}{ccc}
\frac{1}{2} I_{d} & B(\zeta) & 0 \\
0 & G_{2}(\zeta) & C(\zeta) \\
0 & 0 & -i \zeta I_{d}
\end{array}\right), \zeta \in \partial \Delta
$$

where $I_{d}$ denotes the identity matrix of size $d, G_{2}(\zeta)$ is the following invertible matrix of size $2 n$

$$
G_{2}(\zeta)=\left(\begin{array}{cc}
\zeta^{t} A & I_{n} \\
i \zeta^{t} A & -i I_{n}
\end{array}\right)
$$

$B(\zeta)$ is the following matrix of size $d \times 2 n$

$$
B=\left(\begin{array}{cccccc}
-\left(A_{1}\right)^{1} h_{0}(\zeta) & \ldots & -\left(A_{1}\right)^{n} h_{0}(\zeta) & 0 & \ldots & 0 \\
\vdots & & \vdots & \vdots & & \vdots \\
-\left(A_{d}\right)^{1} h_{0}(\zeta) & \ldots & -\left(A_{d}\right)^{n} h_{0}(\zeta) & 0 & \ldots & 0
\end{array}\right)=(1-\zeta) B_{1}
$$


and $C(\zeta)$ is the following matrix of size $2 n \times d$

$$
C=\left(\begin{array}{ccc}
2{ }^{t} h_{0} \overline{\left(A_{1}\right)_{1}} & \ldots & 2{ }^{t} h_{0} \overline{\left(A_{d}\right)_{1}} \\
\vdots & & \vdots \\
2{ }^{t} h_{0} \overline{\left(A_{1}\right)_{n}} & \ldots & 2{ }^{t} h_{0} \overline{\left(A_{d}\right)_{n}} \\
-2 i^{t} h_{0} \overline{\left(A_{1}\right)_{1}} & \ldots & -2 i^{t} h_{0} \overline{\left(A_{d}\right)_{1}} \\
\vdots & & \vdots \\
-2 i^{t} h_{0} \overline{\left(A_{1}\right)_{n}} & \ldots & -2 i^{t} h_{0} \overline{\left(A_{d}\right)_{n}}
\end{array}\right)
$$

where $\left(A_{j}\right)_{l}$ (resp. $\left.\left(A_{j}\right)^{l}\right)$ denotes, for $j=1, \ldots, d$ and $l=1, \ldots, n$, the $l^{\text {th }}$ column (resp. row) of $A_{j}$. The following theorem on the construction of stationary discs with pointwise constraints was obtained in [6].

Theorem 3.1 ([6]). Let $Q \subset \mathbb{C}^{N}$ be a quadric submanifold of real codimension d given by (2.1) and satisfying satisfying $(\mathfrak{t})$. Let $\boldsymbol{f}_{0}=\left(h_{0}, g_{0}, \tilde{h_{0}}, \tilde{g}_{0}\right) \in \mathcal{S}_{0}(Q)$ of the form (2.2). Then there exist open neighborhoods $U$ of $\rho$ in $\left(\mathcal{C}^{4}(\mathbb{B})\right)^{d}$ and $V$ of 0 in $\mathbb{R}^{2 N}$, a real number $\varepsilon>0$ and $a \mathcal{C}^{1}$ map

$$
\mathcal{F}_{0}: U \times V \rightarrow \mathcal{A}
$$

such that:

i. $\mathcal{F}_{0}(\rho, 0)=\boldsymbol{f}_{\mathbf{0}}$,

ii. for all $r \in U$ the map

$$
\mathcal{F}_{0}(r, \cdot): V \rightarrow\left\{\boldsymbol{f} \in \mathcal{S}_{0}(\{r=0\}) \mid\left\|\boldsymbol{f}-\boldsymbol{f}_{\mathbf{0}}\right\|_{\mathcal{A}_{0}^{1, \alpha}}<\varepsilon\right\}
$$

is one-to-one and onto.

iii. the tangent space $T_{\boldsymbol{f}_{\mathbf{0}}} \mathcal{S}_{0}(Q)$ of $\mathcal{S}_{0}(Q)$ at $\boldsymbol{f}_{\mathbf{0}}$ is the kernel of the linear map

$$
2 \Re e[\overline{G(\zeta)} \cdot]:\left(\mathcal{A}_{0}^{1, \alpha}\right)^{2 N} \rightarrow\left(\mathcal{C}_{0}^{1, \alpha}\right)^{2 N}
$$

Remark 3.2. We emphasize that in the previous theorem the model quadric is only supposed to satisfy condition $(\mathfrak{t})$.

3.1. Injectivity of the jet map. The method used to prove Theorem $3.1[6]$ based on the computation of certain integers, namely the partial indices and the Maslov index, implies that discs constructed in Theorem 3.1 are determined by their $k$-jet at $\zeta=1$, where $k$ is the greatest partial index; in the present case $k=2$. For seek of completeness, we refer to the appendix for a proof of that claim. It is important to note that this result only requires the quadric model to satisfy $(\mathfrak{t})$. In this section, we show that in case the quadric model also satisfies ( $\mathfrak{d})$, 1-jet determination can be achieved.

Consider the linear jet map

$$
\mathfrak{j}_{1}:\left(\mathcal{A}^{1, \alpha}\right)^{2 N} \rightarrow \mathbb{C}^{4 N}
$$

mapping $\boldsymbol{f}$ to its 1 -jet at $\zeta=1$, namely

$$
\mathfrak{j}_{1}(\boldsymbol{f})=\left(\boldsymbol{f}(1), \frac{\partial \boldsymbol{f}}{\partial \zeta}(1)\right) .
$$


Proposition 3.3. Let $Q \subset \mathbb{C}^{N}$ be a quadric submanifold of real codimension $d$ given by (2.1), D-nondegenerate at 0 . Consider an initial disc $\boldsymbol{f}_{\mathbf{0}} \in \mathcal{S}_{0}(Q)$ of the form (2.2) where $V$ and $c=\left(c_{1}, \ldots, c_{d}\right)$ are respectively given by $(\mathfrak{d})$ and $(\mathfrak{t})$. Then there exist an open neighborhood $U$ of $\rho$ in $\left(\mathcal{C}^{4}(\mathbb{B})\right)^{d}$ and a positive $\varepsilon$ such that for all $r \in U$ the map $\mathfrak{j}_{1}$ is injective on

$$
\left\{\boldsymbol{f} \in \mathcal{S}_{0}(\{r=0\}) \quad\left\|\boldsymbol{f}-\boldsymbol{f}_{\mathbf{0}}\right\|_{1, \alpha}<\varepsilon\right\} .
$$

In other words, such discs are determined by their 1-jet at 1.

Proof. By the implicit function theorem, it is enough to prove that the restriction of $\mathfrak{j}_{1}$ to the tangent space $T_{f_{0}} \mathcal{S}_{0}(Q)$ of $\mathcal{S}_{0}(Q)$ at the point $\boldsymbol{f}_{\mathbf{0}}$ is injective. By Theorem 3.1, we have to show that the only element of the kernel of the map $2 \Re e[\overline{G(\zeta)} \cdot]:\left(\mathcal{A}_{0}^{1, \alpha}\right)^{2 N} \rightarrow$ $\left(\mathcal{C}_{0}^{1, \alpha}\right)^{2 N}$ with trivial 1-jet at $\zeta=1$ is the trivial disc. Let $\boldsymbol{f}=(1-\zeta)(h, g, \tilde{h}, \tilde{g})$ be such an element. According to the proof of Lemma 3.4 in [6], we have

$$
\left\{\begin{array}{l}
\tilde{g}_{j}=a_{j}-\overline{a_{j}} \zeta, a_{j} \in \mathbb{C} \\
h=A^{-1}(X+Y \zeta), \\
g(\zeta)=-4 \Re e\left(\overline{B_{1}} A^{-1} X\right)+2 \overline{B_{1}} A^{-1} Y-2 \overline{B_{1}} A^{-1} Y \zeta .
\end{array}\right.
$$

where for $k=1, \ldots, n$,

$$
X_{k}=2 \sum_{j=1}^{d}{ }^{t} V \overline{\left(A_{j}\right)_{k}} \Re e\left(a_{j}\right)+\frac{\tilde{y_{k}}}{2}+i \frac{y_{k}}{2} \in \mathbb{C},
$$

$\tilde{y_{k}}, y_{k} \in \mathbb{R}$ and

$$
Y_{k}=-2 \sum_{j=1}^{d}{ }^{t} V \overline{\left(A_{j}\right)_{k}} \overline{a_{j}} \in \mathbb{C} .
$$

Since $\boldsymbol{f}$ has a trivial 1 -jet at 1 , we must have

$$
\left\{\begin{array}{l}
a_{j} \in \mathbb{R} \\
X=-Y \\
\Re e\left(\overline{B_{1}} A^{-1} X\right)=0 .
\end{array}\right.
$$

It follows that

$$
\begin{aligned}
\overline{B_{1}} A^{-1} X & =-\overline{B_{1}} A^{-1} Y \\
& =-2 \underbrace{\left(\begin{array}{ccc}
\overline{\left(A_{1}\right)^{1} V} & \ldots & \overline{\left(A_{1}\right)^{n} V} \\
\vdots & & \vdots \\
\frac{\vdots}{\left(A_{d}\right)^{1} V} & \ldots & \overline{\left(_{d}\right)^{n} V}
\end{array}\right)}_{{ }^{t} \bar{D}} A^{-1} \underbrace{\left(\begin{array}{ccc}
{ }^{t} V \overline{\left(A_{1}\right)_{1}} & \ldots & { }^{t} V \overline{\left(A_{d}\right)_{1}} \\
\vdots & & \vdots \\
{ }^{t} V \overline{\left(A_{1}\right)_{n}} & \ldots & { }^{t} V \overline{\left(A_{d}\right)_{n}}
\end{array}\right)}_{D}\left(\begin{array}{c}
a_{1} \\
\vdots \\
a_{d}
\end{array}\right) \in i \mathbb{R}^{d},
\end{aligned}
$$

and so

$$
\Re e\left({ }^{t} \bar{D} A^{-1} D\right)\left(\begin{array}{c}
a_{1} \\
\vdots \\
a_{d}
\end{array}\right)=0
$$


It follows from condition ( $\mathfrak{d}$ ) that $a_{1}=\ldots=a_{d}=0$. This implies, using (3.1) that $g=\tilde{g}=0$ and $h=0$. Equation (3.2) implies that $y_{k}=\tilde{y}_{k}=0$. Finally an inspection of the proof of Lemma 3.4 in [6] shows that $\tilde{h}=0$.

3.2. Filling an open set in the conormal bundle. In [6], we proved that in case the quadric $Q$ is fully nondegenerate at 0 , the family of centers of stationary discs covers an open set of $\mathbb{C}^{N}$. This relied essentially on the fact that when $V$ is given by condition $(\mathfrak{f})$, the matrix ${ }^{t} \bar{D} A^{-1} D$ defined above in the proof of Proposition 3.3 is invertible, which is no longer the case with condition $(\mathfrak{d})$. Instead of covering an open set of $\mathbb{C}^{N}$, we show that the boundaries of the constructed discs cover an open set in the conormal bundle. Proposition 3.3 is crucial for this approach.

Proposition 3.4. Let $Q \subset \mathbb{C}^{N}$ be a quadric submanifold of real codimension d given by (2.1), D-nondegenerate at 0 . Consider an initial disc $\boldsymbol{f}_{\mathbf{0}} \in \mathcal{S}(Q)$ of the form (2.2) where $V$ and $c=\left(c_{1}, \ldots, c_{d}\right)$ are respectively given by $(\mathfrak{d})$ and $(\mathfrak{t})$. Then there exist an open neighborhood $U$ of $\rho$ in $\left(\mathcal{C}^{4}(\mathbb{B})\right)^{d}$ and a positive $\varepsilon$ such that for all $r \in U$ the set

$$
\left\{e^{-i \theta} \cdot \boldsymbol{f}\left(e^{i \theta}\right) \mid \boldsymbol{f} \in \mathcal{S}_{0}(\{r=0\})\left\|\boldsymbol{f}-\boldsymbol{f}_{\mathbf{0}}\right\|_{1, \alpha}<\varepsilon, 0 \leq \theta<\varepsilon\right\}
$$

covers an open set in the conormal bundle $N^{*}(\{r=0\})$.

The following lemma is a version of Lemma 3, Section 15.5 in 8 .

Lemma 3.5. Let $X_{1}, \ldots, X_{s}$ be linearly independent vectors in $\mathbb{R}^{s}, F: \mathbb{R}^{s} \longrightarrow \mathbb{R}^{s}$ be a continuous map, $S$ be a subset of $\left\{\sum_{j=1}^{s} t_{j} X_{j} \mid t=\left(t_{1}, \ldots, t_{s}\right), t_{j} \geq 0\right\}$. Suppose that there exit $\epsilon>0, \epsilon^{\prime}>0, \eta>0$ such that

i. $\left|F(t)-\sum_{j=1}^{s} t_{j} X_{j}\right| \leq \eta|t|, \quad t=\left(t_{1}, \ldots, t_{s}\right), \quad t_{j} \geq 0, \quad|t| \leq \epsilon^{\prime}$.

ii. For any $t=\left(t_{1}, \ldots, t_{s}\right), t_{j} \geq 0,|t| \leq \epsilon^{\prime}$ such that $t$ has at least one zero component, the line segment between $\sum_{j=1}^{s} t_{j} X_{j}$ and $F(t)$ does not intersect $S \cap B_{\epsilon}$, where $B_{\epsilon}$ is the ball centered at 0 of radius $\epsilon$.

Then the image of $F$ contains $S \cap B_{\epsilon}$.

Proof. The proof of this lemma follows the proof of Lemma 3, Section 15.5 in [8]. It is based on properties of the homology groups (of a sphere), using homotopy and the fact that the set $\left\{\sum_{j=1}^{s} t_{j} X_{j} \mid t=\left(t_{1}, \ldots, t_{s}\right), t_{j} \geq 0\right\} \cap B_{\epsilon^{\prime}}$ is contractile to the origin in $\mathbb{R}^{s}$, while the set $\left\{\sum_{j=1}^{s} t_{j} X_{j}, t=\left(t_{1}, \ldots, t_{s}\right) \mid t_{j} \geq 0\right\} \cap \overline{B_{\epsilon^{\prime}}}$ restricted to the $t$ with at least one zero component is homeomorphic to a $(s-1)$-sphere in $\mathbb{R}^{s}$ with center $x \in S$.

Lemma 3.6. Let $F: \mathbb{R}^{s} \times \mathbb{R} \rightarrow \mathbb{R}^{s}$ be of the form $F(x, \theta)=\theta B(x, \theta)$, where $B: \mathbb{R}^{s} \times \mathbb{R} \rightarrow$ $\mathbb{R}^{s}$ is a continuous function while $B(x, 0): \mathbb{R}^{s} \rightarrow \mathbb{R}^{s}$ is a $\mathcal{C}^{1}$ diffeomorphism at 0 . Then the image of $F$ contains an open set of $\mathbb{R}^{s}$.

Proof. Let $G: \mathbb{R}^{s} \times \mathbb{R} \rightarrow \mathbb{R}^{s} \times \mathbb{R}$ be the map defined by $G(x, \theta)=(F(x, \theta), \theta)$. Using its Taylor expansion, we obtain that for every $\epsilon^{\prime}>0$, there exists $\eta>0$ such that

$$
\left|G(x, \theta)-\theta(B(0), 1)-\sum_{i=1}^{s} \theta x_{i}\left(\frac{\partial B}{\partial x_{i}}(0), 0\right)\right| \leq \eta|(x, \theta)|, \quad|(x, \theta)| \leq \epsilon^{\prime} .
$$

We choose an open cone $\Gamma<\Gamma_{(B(0), 1)\left(\frac{\partial B}{\partial x_{1}}(0), 0\right) \ldots\left(\frac{\partial B}{\partial x_{s}}(0), 0\right)}$, where $\Gamma_{(B(0), 1)\left(\frac{\partial B}{\partial x_{1}}(0), 0\right) \ldots\left(\frac{\partial B}{\partial x_{s}}(0), 0\right)}$ is the cone generated by $(B(0), 1),\left(\frac{\partial B}{\partial x_{1}}(0), 0\right), \ldots,\left(\frac{\partial B}{\partial x_{s}}(0), 0\right)$. Following the proof of Lemma 
3 in [8], there exist $\eta^{\prime}>0$ and $0<\epsilon<\epsilon^{\prime}$, such that the Euclidean distance from $x \in \Gamma \cap B_{\epsilon}$ to the boundary of $\Gamma_{(B(0), 1),\left(\frac{\partial B}{\partial x_{1}}(0), 0\right) \ldots\left(\frac{\partial B}{\partial x_{s}}(0), 0\right)} \cap B_{\epsilon^{\prime}}$ is greater than $\eta^{\prime}|x|$. Choosing $\epsilon^{\prime}$ small enough in order to have $\eta<<\eta^{\prime}$ in (3.3), the assumptions of Lemma 3.5 will be satisfied. Therefore we obtain that the image under $G$ contains $\Gamma \cap B_{\epsilon}$, and hence the image under $F$ contains an open set of $\mathbb{R}^{s}$.

In the following proof, we will be using the notation

$$
u(\Theta)=\left(u_{1}\left(\theta_{1}\right), \ldots, u_{4 N}\left(\theta_{4 N}\right)\right)
$$

for a vector valued function $u: \mathbb{R} \rightarrow \mathbb{R}^{4 N}$ and $\Theta=\left(\theta_{1}, \ldots, \theta_{4 N}\right) \in \mathbb{R}^{4 N}$.

Proof of Proposition 3.4. Consider the map

$$
\Psi_{r}:\left\{\boldsymbol{f} \in \mathcal{S}_{0}(\{r=0\}) \mid\left\|\boldsymbol{f}-\boldsymbol{f}_{\mathbf{0}}\right\|_{1, \alpha}<\varepsilon\right\} \times[0, \varepsilon) \rightarrow N^{*}(\{r=0\})
$$

defined by

$$
\Psi_{r}(\boldsymbol{f}, \theta)=e^{-i \theta} \cdot \boldsymbol{f}\left(e^{i \theta}\right)=\left(f\left(e^{i \theta}\right), e^{-i \theta} \tilde{f}\left(e^{i \theta}\right)\right)
$$

In order to prove the proposition, we will show that the image of $\Psi_{\rho}$ covers an open set by using Lemma 3.6. We also choose coordinate in $N^{*}(Q)$ such that $\boldsymbol{f}_{\mathbf{0}}(1)=0$. Note that we write

$$
\Psi_{\rho}(\boldsymbol{f}, \theta)=\boldsymbol{f}(1)+\theta \frac{d}{d \theta}\left(e^{-i \theta} \cdot \boldsymbol{f}\left(e^{i \theta}\right)\right)(\Theta)=\theta \frac{d}{d \theta}\left(e^{-i \theta} \cdot \boldsymbol{f}\left(e^{i \theta}\right)\right)(\Theta)
$$

for some $\Theta=\left(\theta_{1}, \ldots, \theta_{4 N}\right) \in \mathbb{R}^{4 N}$ with $0<\theta_{j}<\theta, j=1 \ldots, 4 N$, and hence is of the form as in Lemma 3.6. We need to prove that the map defined on $T_{f_{0}} \mathcal{S}_{0}(Q)$

$$
\boldsymbol{f} \mapsto \frac{\partial \Psi_{\rho}(\boldsymbol{f}, 0)}{\partial \theta}=\frac{d}{d \theta}\left(e^{-i \theta} \cdot \boldsymbol{f}\left(e^{i \theta}\right)\right)_{\left.\right|_{\theta=0}}=\left(i f^{\prime}(1), i \tilde{f}^{\prime}(1)\right)
$$

is a diffeomorphism onto an open set in $T_{f_{0}(1)} N^{*} Q$. According to Proposition 3.3, the map $\boldsymbol{f} \mapsto \boldsymbol{f}^{\prime}(1)$ is injective and so is $\boldsymbol{f} \mapsto \frac{d}{d \theta}\left(e^{-i \theta} \cdot \boldsymbol{f}\left(e^{i \theta}\right)\right)_{\left.\right|_{\theta=0}}$. It follows from Lemma 3.6 that the image of $\Psi_{\rho}$ covers an open set in $N^{*}(Q)$.

3.3. Jet determination of CR automorphisms. Let $k$ be a positive integer. Let $M \subset \mathbb{C}^{N}$ be a $\mathcal{C}^{4}$ generic real submanifold and let $p \in M$. We denote by $A u t^{k}(M, p)$ the set of germs at $p$ of $\mathrm{CR}$ automorphisms $F$ of $M$ of class $\mathcal{C}^{k}$; in particular we have $F(p)=p$ and $F(M) \subset M$.

Theorem 3.7. Let $M \subset \mathbb{C}^{N}$ be a $\mathcal{C}^{4}$ generic real submanifold. Assume that $M$ is $\mathfrak{D}$ nondegenerate at $p \in M$. Then elements of $\operatorname{Aut}^{3}(M, p)$ are uniquely determined by their 2 -jet at $p$.

We sketch the proof which is nearly identical to the one of Theorem 4.1 in [6]. Let $F$ be a $\mathrm{CR}$ automorphism class $\mathcal{C}^{3}$ of $M$, fixing $p=0$. Let $\boldsymbol{f}_{\mathbf{0}} \in \mathcal{S}_{0}(Q)$ be a a lift of a stationary disc for $Q$ of the form (2.2), where $Q$ is the model quadric approximating $M$. Let $q \in N^{*} M$ close to $\boldsymbol{f}_{\mathbf{0}}(1)$ and let $\boldsymbol{f} \in \mathcal{S}_{0}(M)$ be a lift of stationary disc for $M$ obtained in Theorem 3.1 with $q=e^{-i \theta} \cdot \boldsymbol{f}\left(e^{i \theta}\right)=\left(f\left(e^{i \theta}\right), e^{-i \theta} \tilde{f}\left(e^{i \theta}\right)\right)$. Since $F$ has a trivial 2-jet at $p=0$, the discs $F_{*} \boldsymbol{f}(\zeta)=\left(F \circ f(\zeta), \tilde{f}(\zeta)\left(d_{f(\zeta)} F\right)^{-1}\right)$ and $\boldsymbol{f}$ have the same 1-jet at $\zeta=1$ and therefore coincide according to Proposition 3.3. It follows that the lift of 
$F$, still denoted by $F$ satisfies $F(q)=q$. Since such points cover an open set in $N^{*} M$ by Proposition 3.4, the map $F$ is the identity.

\section{APPENDIX: JeT DETERMINATION OF STATIONARY DiSCS}

Consider the linear jet map

$$
\mathfrak{j}_{2}:\left(\mathcal{A}^{2, \alpha}\right)^{2 N} \rightarrow \mathbb{C}^{6 N}
$$

mapping $\boldsymbol{f}$ to its 2 -jet at $\zeta=1$, namely

$$
\mathfrak{j}_{2}(\boldsymbol{f})=\left(\boldsymbol{f}(1), \frac{\partial \boldsymbol{f}}{\partial \zeta}(1), \frac{\partial^{2} \boldsymbol{f}}{\partial \zeta^{2}}(1)\right) \in \mathbb{C}^{6 N} .
$$

Let $M$ be a $\mathcal{C}^{4}$ generic real submanifold of $\mathbb{C}^{N}$ of codimension $d$ given by (1.1) and satisfying $(\mathfrak{t})$. Consider a lift of stationary disc $\boldsymbol{f}=(f, \tilde{f})$ for $M$ satisfying $\boldsymbol{f}(1)=$ $\left(0, \sum_{j=1}^{d} c_{j} \partial r_{j}(0)\right)$ where $\sum_{j=1}^{d} c_{j} A_{j}$ is invertible. It follows from Proposition 2.2 and from Chirka (Theorem 33 in [10]) that such discs are of class $\mathcal{C}^{2, \alpha}$ for any $0<\alpha<1$ near $\zeta=1$. Therefore the map $\mathfrak{j}_{2}$ is well defined on the space of lifts of stationary discs.

Proposition 3.8. Let $Q \subset \mathbb{C}^{N}$ be a quadric submanifold of real codimension $d$ given by (2.1), satisfying $(\mathfrak{t})$. Consider an initial disc $\boldsymbol{f}_{\mathbf{0}} \in \mathcal{S}_{0}(Q)$ of the form (2.2) where $c=\left(c_{1}, \ldots, c_{d}\right)$ is given by $(\mathfrak{t})$. Then there exist an open neighborhood $U$ of $\rho$ in $\left(\mathcal{C}^{4}(\mathbb{B})\right)^{d}$ and a positive $\varepsilon$ such that for all $r \in U$ the map $\mathfrak{j}_{2}$ is injective on $\{\boldsymbol{f} \in \mathcal{S}(\{r=0\}) \mid \| \boldsymbol{f}-$ $\left.\boldsymbol{f}_{\mathbf{0}} \|_{1, \alpha}<\varepsilon\right\}$; in other words, such discs are determined by their 2-jet at 1.

Proof. By the implicit function theorem, it is enough to prove that the restriction of $\mathfrak{j}_{2}$ to the tangent space $T_{\boldsymbol{f}_{0}} \mathcal{S}_{0}(Q)$ of $\mathcal{S}_{0}(Q)$ at the point $\boldsymbol{f}_{\mathbf{0}}$ is injective. By Theorem [3.1, we have to show that the only element of the kernel of the map $2 \Re e[\overline{G(\zeta)} \cdot]:\left(\mathcal{A}_{0}^{1, \alpha}\right)^{2 N} \rightarrow$ $\left(\mathcal{C}_{0}^{1, \alpha}\right)^{2 N}$ with trivial 2 -jet at $\zeta=1$ is the trivial disc. Let $\boldsymbol{f}$ be such an element. We have

$$
\overline{G(\zeta)} \boldsymbol{f}+G(\zeta) \overline{\boldsymbol{f}}=0
$$

The last $d$ rows of that equation are of the form

$$
i \bar{\zeta} \tilde{g}_{j}(\zeta)-i \zeta \overline{\tilde{g}_{j}(\zeta)}=0, j=1, \ldots, n,
$$

from which it follows that

$$
\tilde{g}_{j}=a_{j}+b_{j} \zeta+\overline{a_{j}} \zeta^{2}, a_{j} \in \mathbb{C}, b_{j} \in \mathbb{R} .
$$

Since $\tilde{g}_{j}$ has a trivial two jet it follows that $\tilde{g}_{j} \equiv 0$. Solving the system backward, the previous $2 n$ rows of Equation (3.4) are of the form

$$
\overline{G_{2}(\zeta)}\left(\begin{array}{l}
h \\
\tilde{h}
\end{array}\right)+G_{2}(\zeta)\left(\frac{\bar{h}}{\tilde{h}}\right)=0 .
$$

The fact that $h \equiv \tilde{h} \equiv 0$ is a consequence of the fact that all partial indices of $-\overline{G_{2}^{-1}} G_{2}$ are 1. Indeed, following the operations that lead to the introduction of $G_{2}^{b}$ (see Equation 
(2.11) in [6]), we end up with $n$ systems

$$
\overline{R(\zeta)}\left(\begin{array}{c}
h_{k} \\
\tilde{h_{k}}
\end{array}\right)+R(\zeta)\left(\overline{\frac{h_{k}}{\tilde{h_{k}}}}\right)=0 .
$$

with the abuse of notation $h={ }^{t} A h$ - A being invertible -, or equivalently

$$
\left(\begin{array}{c}
h_{k} \\
\tilde{h_{k}}
\end{array}\right)=-\overline{R(\zeta)^{-1}} R(\zeta)\left(\overline{\frac{\overline{h_{k}}}{\tilde{h_{k}}}}\right)=-\left(\begin{array}{ll}
0 & \zeta \\
\zeta & 0
\end{array}\right)\left(\overline{\frac{h_{k}}{\tilde{h_{k}}}}\right)=0 .
$$

According to Lemma 5.1 in [16] we can write

$$
-\left(\begin{array}{ll}
0 & \zeta \\
\zeta & 0
\end{array}\right)=\Theta^{-1}\left(\begin{array}{ll}
\zeta & 0 \\
0 & \zeta
\end{array}\right) \bar{\Theta}
$$

where $\Theta: \bar{\Delta} \rightarrow G l_{2}(\mathbb{C})$ is a smooth map holomorphic on $\Delta$; more explicitly one can take for instance

$$
\Theta=\left(\begin{array}{cc}
1 & -1 \\
i & i
\end{array}\right)
$$

Therefore

$$
\Theta\left(\begin{array}{c}
h_{k} \\
\tilde{h_{k}}
\end{array}\right)=\left(\begin{array}{ll}
\zeta & 0 \\
0 & \zeta
\end{array}\right) \overline{\Theta\left(\begin{array}{c}
h_{k} \\
h_{k}
\end{array}\right)}
$$

It follows that each of the two components of $\Theta\left(\begin{array}{c}h_{k} \\ \tilde{h_{k}}\end{array}\right)$ is a polynomial of degree one, and so are $h_{k}$ and $\tilde{h_{k}}$ (due to the explicit form of $\Theta$ ). Since they have a trivial 2-jet at 1 , this implies that $h_{k} \equiv \tilde{h_{k}} \equiv 0, k=1, \ldots, n$, and thus $h \equiv \tilde{h} \equiv 0$. Finally the first $d$ equations implies directly hat $g \equiv 0$.

\section{REFERENCES}

[1] M.S. Baoudendi, P. Ebenfelt, L.P. Rothschild, CR automorphisms of real analytic CR manifolds in complex space, Comm. Anal. Geom. 6 (1998), 291-315.

[2] M.S. Baouendi, N. Mir, L.P. Rothschild, Reflection Ideals and mappings between generic submanifolds in complex space, J. Geom. Anal. 12 (2002), 543-580.

[3] M.S. Baouendi, L.P. Rothschild, J.-M. Trépreau On the geometry of analytic discs attached to real manifolds, J. Differential Geom. 39 (1994), 379-405.

[4] V.K. Beloshapka, Finite dimensionality of the group of automorphisms of a real-analytic surface, Math. USSR Izvestiya 32 (1989), 239-242.

[5] F. Bertrand, L. Blanc-Centi, Stationary holomorphic discs and finite jet determination problems, Math. Ann. 358 (2014), 477-509.

[6] F. Bertrand, L. Blanc-Centi, F. Meylan, Stationary discs and finite jet determination for nondegenerate generic real submanifolds, Adv. Math. 343 (2019), 910-934.

[7] F. Bertrand, G. Della Sala, B. Lamel, Jet determination of smooth CR automorphisms and generalized stationary discs, Math. Z. 294 (2020), 1611-1634.

[8] Boggess Albert, CR Manifolds and the Tangential Cauchy-Riemann Complex, Studies in Advanced Mathematics (1991).

[9] S.S. Chern, J.K. Moser, Real hypersurfaces in complex manifolds, Acta math. 133 (1975), 219271.

[10] E.M. Chirka, Regularity of the boundaries of analytic sets, Mat. Sb. 45 (1983), 291-336. 
[11] E. Cartan, Sur la géométrie pseudo-conforme des hypersurfaces de deux variables complexes, I. Ann. Math. Pura Appl. 11 (1932), 17-90 (Euvres complètes, Part. II, Gauthier-Villars, 1952, 1231-1304); II. Ann. Sc. Norm. Sup. Pisa 1 (1932), 333-354 (CEuvres complètes, Part. III, Gauthier-Villars, 1952, 1217-1238).

[12] P. Ebenfelt, Finite jet determination of holomorphic mappings at the boundary, Asian J. Math. 5 (2001), 637-662.

[13] P. Ebenfelt, B. Lamel, Finite jet determination of CR embeddings, J. Geom. Anal. 14 (2004), 241-265.

[14] P. Ebenfelt, B. Lamel, D. Zaitsev, Finite jet determination of local analytic CR automorphisms and their parametrization by 2-jets in the finite type case, Geom. Funct. Anal. 13 (2003), 546573.

[15] F. Forstnerič, Analytic disks with boundaries in a maximal real submanifold of $\mathbb{C}^{2}$, Ann. Inst. Fourier 37 (1987), 1-44.

[16] J. Globevnik, Perturbation by analytic discs along maximal real submanifolds of $\mathbb{C}^{N}$, Math. Z. 217 (1994), 287-316.

[17] X. Huang, A non-degeneracy property of extremal mappings and iterates of holomorphic selfmappings, Ann. Scuola Norm. Sup. Pisa Cl. Sci. (4) 21 (1994), 399-419.

[18] R. Juhlin, Determination of formal CR mappings by a finite jet, Adv. Math. 222 (2009), 16111648 .

[19] R. Juhlin, B. Lamel, Automorphism groups of minimal real-analytic CR manifolds, J. Eur. Math. Soc. (JEMS) 15 (2013), 509-537.

[20] S.-Y. Kim, D. Zaitsev, Equivalence and embedding problems for CR-structures of any codimension, Topology 44 (2005), 557-584.

[21] M. Kolár, F. Meylan, D. Zaitsev, Chern-Moser operators and polynomial models in CR geometry, Adv. Math. 263 (2014), 321-356.

[22] B. Lamel, N. Mir, Finite jet determination of CR mappings, Adv. Math. 216 (2007), 153-177.

[23] L. Lempert, La métrique de Kobayashi et la représentation des domaines sur la boule, Bull. Soc. Math. France 109 (1981), 427-474.

[24] F. Meylan, A counterexample to the 2-jet determination Chern-Moser Theorem in higher codimension, preprint, arXiv:2003.11783.

[25] N. Mir, D. Zaitsev, Unique jet determination and extension of germs of CR maps into spheres, to appear in Trans. Amer. Math. Soc., arXiv:2008.07194.

[26] M.-Y. Pang, Smoothness of the Kobayashi metric of nonconvex domains, Internat. J. Math. 4 (1993), 953-987.

[27] A. Sukhov, A. Tumanov, Stationary discs and geometry of CR manifolds of codimension two, Internat. J. Math. 12 (2001), 877-890.

[28] N. Tanaka, On the pseudo-conformal geometry of hypersurfaces of the space of $n$ complex variables, J. Math. Soc. Japan 14 (1962), 397-429.

[29] A. Tumanov, Extension of CR-functions into a wedge from a manifold of finite type, (Russian) Mat. Sb. (N.S.) 136 (178) (1988), 128-139; translation in Math. USSR-Sb. 64 (1989), 129-140.

[30] A. Tumanov, Extremal discs and the regularity of $C R$ mappings in higher codimension, Amer. J. Math. 123 (2001), 445-473.

[31] A. Tumanov, Stationary Discs and finite jet determination for CR mappings in higher codimension, Adv. Math. 371 (2020), 107254, 11 pp.

[32] S. Webster, On the reflection principle in several complex variables, Proc. Amer. Math. Soc. 71 (1978), 26-28.

[33] D. Zaitsev, Germs of local automorphisms of real analytic CR structures and analytic dependence on the $k$-jets, Math. Res. Lett. 4 (1997), 1-20. 
Florian Bertrand

Department of Mathematics,

American University of Beirut, Beirut, Lebanon

E-mail address: fb31@aub.edu.lb

Francine Meylan

Department of Mathematics

University of Fribourg, CH 1700 Perolles, Fribourg

E-mail address: francine.meylan@unifr.ch 